This is the peer reviewed version of the following article: A.-L. Sahlberg, D. Hot, J. Kiefer, M. Aldén, and Z. Li, "Mid-infrared laser-induced thermal grating spectroscopy in flames", Proceedings of the Combustion Institute, 36, 4515-4523, (2016), which has been published in final form at https://doi.org/10.1016/j.proci.2016.07.017. 


\section{Mid-infrared laser-induced thermal grating spectroscopy in flames}

Anna-Lena Sahlberg ${ }^{1}$, Dina Hot $^{1}$, Johannes Kiefer ${ }^{2, *}$, Marcus Aldén ${ }^{1}$ and Zhongshan Li $^{1}$

${ }^{1}$ Division of Combustion Physics, Lund University, P.O. Box 118, S221 00 Lund, Sweden

${ }^{2}$ Technische Thermodynamik, Universität Bremen, Badgasteiner Str. 1, 28359 Bremen,

Germany

*Corresponding author: Johannes Kiefer

Technische Thermodynamik, Universität Bremen

Badgasteiner Str. 1, 28359 Bremen, Germany

Fax: $\quad+4942121864771$

Phone: +49 42121864777

Email: jkiefer@uni-bremen.de

Colloquium: DIAGNOSTICS

Total length of the manuscript (Method 1): 6200 (max. 6200)

Word equivalent lengths: Main text: 4121

Equation: $\quad 30$

References: 577

Fig. 1: $\quad 175$

Fig. 2: $\quad 177$

Fig. 3: 199

Fig. 4: 220

Fig. 5: 269

Fig. 6: 173

Fig. 7: 259 


\section{Abstract}

For the first time, laser-induced thermal grating spectroscopy (LITGS) in the spectral range around $3 \mu \mathrm{m}$ is demonstrated as a versatile diagnostic tool. This spectral region is of particular interest in combustion diagnostics as many relevant species such as hydrocarbons and water exhibit fundamental vibrational modes and hence can be probed with high sensitivity. Another benefit of the IR-LITGS is that it allows performing spectroscopy in the infrared combined with signal detection in the visible. Hence, the strong thermal radiation inherent in flames does not represent an interference. As the first step, we present the application of IR-LITGS to cold gas flows, where traces of ethylene and water vapor are detected. The time-resolved LITGS signals, which can be acquired in a single laser shot, are rich in information and allow deriving temperature and to some extend chemical composition. In the second step, the IR-LITGS technique is applied to ethylene/air flames stabilized on a flat flame burner. A proof-of-concept study is carried out, in which the temperature is determined in the burned region of flames with systematically varied equivalence ratio $(0.72<\Phi<2.57)$. Moreover, in a highly sooty flame, LITGS signals were recorded as a function of height above the burner and allowed the determination of the temperature profile. The proposed IR-LITGS method has the potential for enabling single-shot measurements of several parameters at a time. Its applicability to sooty flame environments opens up new opportunities to study the complex formation of carbonaceous particles in flames.

Keywords: laser-induced thermal grating spectroscopy, mid-infrared, ethylene/air flame, temperature, concentration 


\section{Introduction}

A major task in combustion research is to experimentally validate combustion models and hence there is a need for diagnostic methods [1]. Spectroscopic techniques such as Raman scattering, laser-induced fluorescence, and coherent anti-Stokes Raman scattering (CARS) have been developed since the 1960s to obtain information about temperature and species concentrations in combustion environments in a nonintrusive manner. High accuracy and precision as well as spatial and temporal resolution are required in the acquisition of data appropriate for model evaluation.

The application of laser diagnostics to combustion systems is most useful when several parameters can be measured simultaneously. Temperature, a key parameter for flame characterization, should always be included in the list of requirements. However, due to the particularly harsh environment in sooty flames of practical interest, most conventional techniques reach their limits. Line-of-sight methods like absorption or emission spectroscopy do not provide sufficient spatial resolution. Rayleigh scattering, Raman spectroscopy and laserinduced fluorescence may suffer from the presence of the particles. In order to overcome these problems, nonlinear optical methods have proved to be most suited [2, 3].

In particular, CARS for temperature and selected species measurements have frequently been used [4-6]. Especially in heavily sooting environments, CARS has demonstrated its advantages [7-10]. Laser-induced thermal grating scattering (LITGS) is another promising candidate for flame diagnostics. Time-resolved LITGS signals are rich in information, e.g. regarding the temperature, chemical composition, quenching effects and the thermo-physical properties of the probed gas volume. In principle, laser-induced gratings (LIGs) are transient spatially periodic modulations of the refractive index. In a typical LITGS experiment, two coherent pump beams from a pulsed laser are overlapped and create an interference pattern in the intersection region. Thermalization of the energy conveyed to the absorbing species of the 
medium by the pump radiation result in transient density, and thus refractive index spatial modulations [3]. For completeness, we note that electrostriction is also taking place, but it can usually be neglected in the presence of strong absorption. A small fraction of a continuouswave probe laser beam is Bragg diffracted by the LIG and represents the signal, which is detected with high temporal resolution to allow monitoring the dynamics of the transient grating [3].

To date laser-induced grating methods have been applied to combustion systems to a limited extent. Most of the studies used LIG techniques in non-sooty flames. For example, hydrogen flames were examined by Williams et al. [11]. They used a dye laser at $308 \mathrm{~nm}$ to excited $\mathrm{OH}$ radicals and employed a pulsed probe laser (532 nm). Fourkas et al. employed two picosecond lasers at 589.6 and $589.0 \mathrm{~nm}$ to resonantly pump and probe sodium atoms seeded into hydrogen and methane flames [12]. Hell et al. [13] utilized the absorption band of water around $1 \mu \mathrm{m}$ to obtain laser-induced thermal gratings in a $\mathrm{H}_{2}$ /air flame using the fundamental output of a Nd:YAG laser. The gratings were probed with a pulsed laser at $532 \mathrm{~nm}$. A number of different pump-probe schemes were also applied to lean and stoichiometric methane/air flames. Latzel et al. [14] excited $\mathrm{OH}$ radicals at $307 \mathrm{~nm}$ and used a cw Argon ion laser (488 nm) as a probe. The absorption of water and $\mathrm{OH}$ molecules in the near-infrared was utilized by Hart et al. [15], Hemmerling et al. [16], and Stampanoni-Panariello et al. [17]. They employed pump lasers at 720, 820, and $1064 \mathrm{~nm}$, respectively. The latter excitation wavelength was also used in a study by Hemmerling and Kozlov [18], who demonstrated a single-laser scheme for LIG experiments. The mid-infrared radiation from a $\mathrm{CO}_{2}$ laser was employed by Gutfleisch et al. [19]. The long and non-Gaussian pulses from the $\mathrm{CO}_{2}$ laser, however, did not allow the observation of the typical acoustic oscillations of transient LIG signals generated with nano- or picosecond pulses.

Sooty flames have been studied with LIG techniques even less. As early as 1993 did Hemmerling and Stampanoni-Panariello apply a two-dimensional LIG method to image a sooty 
acetylene/air flame [20]. Their setup, however, did not allow recordings of time-resolved data and hence it was not possible to make use of the rich information contained in the grating dynamics. Such time-resolved LIG signals from sooty flames were studied by Brown and coworkers [21, 22]. They utilized the acoustic modulations during the first 100 ns after the pump pulses to derive temperature information in ethylene/air flames [21]. The pump laser provided $50 \mathrm{~mJ}$ pulses at $532 \mathrm{~nm}$, and the cw probe laser delivered $500 \mathrm{~mW}$ at $488 \mathrm{~nm}$. In the presence of soot particles, an increase in the signal intensity was observed due to the absorption of pump radiation by the particles. In a follow-up study they used $100 \mathrm{~mJ}$ pump pulse energy (532 nm) and up to $1.5 \mathrm{~W}$ cw power (514.5 nm) as a probe.

In most applications of laser-induced grating methods to flames to date, relatively high laser pulse energies have been used. The reason for this may be that excitation was often made in the visible at $532 \mathrm{~nm}$. This wavelength is not absorbed by the molecules in a flame and hence the signal in non-sooty regions was a result of electrostriction, which is a relatively weak effect compared to thermalization. Electrostrictive LIG signals are dominated by the overall acoustic properties (speed of sound and sound attenuation), and consequently the content of information is significantly lower than in LITGS signals. For multi-parameter measurements, laser-induced thermal gratings seem to be more promising. In an optimal case, these thermal gratings are produced by excitation of species that naturally occur in a flame in order to avoid the need for seeding a tracer like acetone [23] or sodium [12]. Molecular tracers may be oxidized in the reaction zone and hence measurements are only possible in the unburnt gas region of a flame. Utilizing combustion intermediates like $\mathrm{OH}[11,14]$ or products like water $[13,15,16]$ is an option, but again, only limited regions can be studied. A suitable alternative is to do excitation at around $3 \mu \mathrm{m}$, where many combustion-relevant species absorb via fundamental rovibrational transitions [24]. This is true for hydrocarbons, water, and even for soot particles. Hence it seems promising to apply IR-LIGS to flames. 
In this work, we demonstrate the first application of IR-LITGS in lean and rich $\mathrm{C}_{2} \mathrm{H}_{4} /$ air flames. For this purpose, tunable radiation from a pulsed infrared laser is employed to produce a transient laser-induced grating inside McKenna-type flames. A $457 \mathrm{~nm}$ cw laser is used to probe the dynamics of the grating. In a first series of measurements, the IR laser was tuned across a large spectral window around $3 \mu \mathrm{m}$ in order to record the spectra in different gas flows and flames. With the knowledge of the spectroscopy, LITGS signals were acquired systematically at varied locations in various ethylene/air flames stabilized with a bluff body mounted above the burner.

\section{Experimental setup}

The IR-LITGS measurements were performed in atmospheric laminar ethylene/air flames with equivalence ratio ranging from $\Phi=0.72$ to $\Phi=2.57$ on a McKenna-type burner with a plug diameter of $48 \mathrm{~mm}$. The water-cooled burner head was utilized to provide a flat premixed flame with a bluff body stabilizer mounted $14 \mathrm{~mm}$ above the burner surface. The relative flows of ethylene and air were controlled with mass flow controllers (Bronkhorst).

A schematic view of the experimental setup is illustrated in Fig. 1. The laser system has been described in more detail elsewhere [25] and thus only a brief description is provided here. The mid-IR laser radiation around $3 \mu \mathrm{m}$ was generated by a laser system consisting of an injectionseeded Nd:YAG laser (Spectra Physics, PRO 290-10), a dye laser (Sirah, PRSC-D-18) and a frequency mixing unit. The fundamental beam $(1064 \mathrm{~nm})$ of the Nd:YAG laser was frequency doubled to $532 \mathrm{~nm}$ to pump the tunable dye laser operating with LDS 798 dye. A part of the residual $1064 \mathrm{~nm}$ beam from the $\mathrm{Nd}$ :YAG laser was frequency mixed in a $\mathrm{LiNbO}_{3}$ crystal with the dye laser output tuned to $806 \mathrm{~nm}$, resulting in IR laser pulses. This IR laser beam was further amplified in a second $\mathrm{LiNbO}_{3}$ crystal pumped with another part of the residual $1064 \mathrm{~nm}$ beam 
to produce pulsed tunable mid-IR laser light with pulse energies around $7 \mathrm{~mJ}$ and pulse durations of 3-4 ns. The linewidth of the IR laser has been measured to be $0.025 \mathrm{~cm}^{-1}$ in previous work [26]. The IR laser beam was guided through a telescope arrangement (L1 and L2) to obtain a beam size of approximately $2 \mathrm{~mm}$. A HeNe laser beam was spatially overlapped with the IR beam through a dichroic mirror to facilitate the optical alignment. The IR beam was split into two parallel beams with equal intensity using a specially coated $\mathrm{CaF}_{2}$ plate (BS, 50/50), resulting in the two pump beams used in IR-LITGS. A cw solid state laser (Laserglow Tech., LRS-0457) with a wavelength of $457 \mathrm{~nm}$ and an output power of $330 \mathrm{~mW}$ was used as probe laser. The pump beams were focused by a $\mathrm{CaF}_{2}$ lens (L3) with $f=300 \mathrm{~mm}$ over the burner, resulting in a crossing angle of $5.65^{\circ}$ and a grating spacing of $31 \mu \mathrm{m}$ with an estimated interaction length of $14 \mathrm{~mm}$. The phase-matching was achieved in a planar BOXCARS geometry and thus, the probe laser was focused into the crossing point with the same lens. After the measurement region, the IR-LITGS signal beam was separated from the pump and probe beams using an aperture and interference filter (IF, $457.9 \pm 10 \mathrm{~nm}$ ) and was directed to a photomultiplier tube (PMT, Hamamatsu H6780-04) connected to an oscilloscope (LeCroy WaveRunner 6100, $1 \mathrm{GHz}$ ).

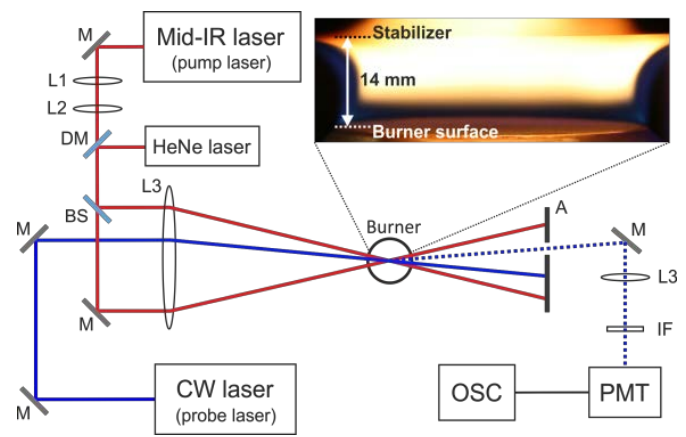

Figure 1: Schematic view of the IR-LITGS setup. M: gold or aluminum mirror, DM: dichroic mirror, BS: $\mathrm{CaF}_{2}$ beam splitter (50/50), L1: CaF 2 lens, $f=500 \mathrm{~mm}, \mathrm{L2}: \mathrm{CaF}_{2}$ lens, $f=-200 \mathrm{~mm}$, L3: $\mathrm{CaF}_{2}$ lens, $f=300 \mathrm{~mm}, \mathrm{~A}$ : aperture, IF: interference filter $457.9 \pm 10 \mathrm{~nm}, \mathrm{PMT}$ : 
photomultiplier tube, OSC: oscilloscope. The photograph shows the laminar $\mathrm{C}_{2} \mathrm{H}_{4} /$ air flame at $\Phi=2.57$.

\section{Results \& Discussion}

The theory of LITGS has been presented several times (see, e.g. [3, 16, 27, 28]) and only a brief description is included here. Two pump beams of wavelength $\lambda_{\text {pump }}$ crossing at an angle $\theta$ create a laser-induced thermal grating (LITG) with grating spacing $\Lambda=\lambda_{\text {pump }} /(2 \sin (\theta / 2))$. The dynamics of the LITG can be studied by detecting the time-resolved probe beam scattering. The rise time of the signal depends on the speed of the energy transfer in the medium, and the signal decays exponentially due to thermal diffusion. In addition, the fast changes of the temperature during the formation of the LITG will create a rapid density modulation. This leads to two acoustic waves propagating perpendicular to the fringe plane in opposite direction; consequently the formation of a standing wave. The frequency of the acoustic signal is $f_{\text {osc }}=$ $v_{s} / \Lambda$, where $v_{s}$ is the speed of sound in the gas. This acoustic contribution will appear as an oscillation of the signal intensity superimposed on the signal from the stationary temperature grating. This acoustic wave modulation will decay as the waves travel out of the grating area. Sound attenuation by viscous damping can normally be neglected.

The temperature $T$ for an ideal gas can be calculated as

$$
T=\frac{v_{s}^{2}}{R_{u}\left(\gamma / M_{g a s}\right)}
$$

where $\gamma$ is the specific heat ratio, $R_{u}$ is the universal gas constant and $M_{\text {gas }}$ is the molecular weight of the gas. Thus, the oscillation frequency in the LITGS signal can be used to calculate 
the gas temperature, if the gas composition and the relevant gas constants of the medium are known.

\subsection{LITGS in non-reacting flows}

Figure 2 shows the temporal shape of a LITGS signal recorded in a room temperature gas flow of $2.6 \% \mathrm{C}_{2} \mathrm{H}_{4}$ diluted in $\mathrm{N}_{2}$. The pump laser wavelength was $3.125 \mu \mathrm{m}\left(3200 \mathrm{~cm}^{-1}\right)$ and the curve is an average of 500 shots. The frequency of the oscillation, determined by Fourier analysis of the signal, is $11 \mathrm{MHz}$. This value was used for calibration of the crossing angle between the pump beams, to increase the accuracy of the subsequent temperature measurements in the flames. By fitting a single-exponential function to the decaying part of the signal, the decay time $\tau_{d}$ was found to be $1.23 \mu$ s. The red curve in Fig. 2 is the $532 \mathrm{~nm} \mathrm{Nd:YAG} \mathrm{laser}$ pulse recorded with a photodiode. This is taken as a reference for the pump laser timing. The mid-IR pump laser pulses were delayed by a short time ( 10 ns) compared to the $532 \mathrm{~nm}$ pulse. This delay has been compensated for in the figures.

The inset in Fig. 2 shows a LITGS excitation scan in the interval 3200-3240 $\mathrm{cm}^{-1}$. Each point in the excitation scan represents the integrated area under the LITGS signal between 0 and 5 $\mu$ s. The excitation scan was recorded with $\sim 1.5 \mathrm{~mJ}$ pump laser energy, to avoid saturation broadening in the recorded spectrum.

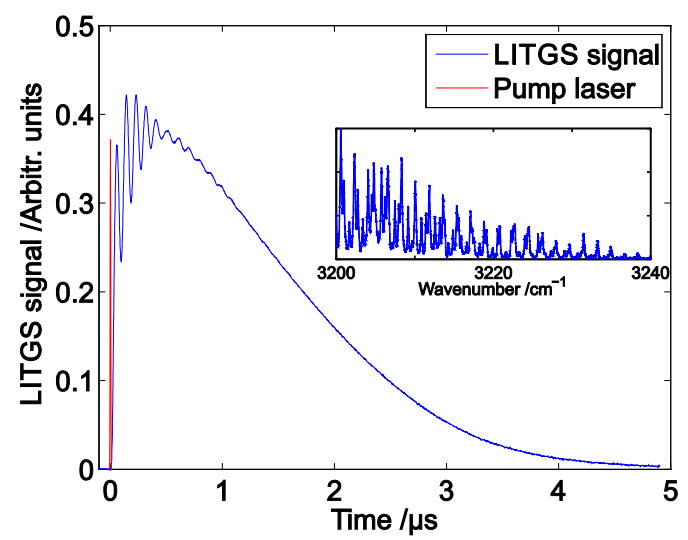


Figure 2. LITGS temporal shape at $3200 \mathrm{~cm}^{-1}$ in a gas flow of $2.6 \% \mathrm{C}_{2} \mathrm{H}_{4}$ diluted in $\mathrm{N}_{2}$ at 296 K. The red curve shows the timing of the pump laser pulses. Inset: LITGS excitation scan 3200$3240 \mathrm{~cm}^{-1}$.

During the experiments it was found that the LITGS signal is very sensitive to water lines in this spectral region. Even diffusion of a small amount of ambient water vapor into the gas flow caused a strong interfering signal. This is remarkable as the water lines in this spectral range possess low absorption cross sections. To test the sensitivity of LITGS to water lines, we recorded a LITGS excitation scan in the ambient air. The water concentration in the air was estimated to be $\sim 0.5 \%$ from the humidity and temperature in the lab. Figure 3 shows the LITGS excitation scan in the interval 3200-3300 $\mathrm{cm}^{-1}$ compared with a simulation of the $\mathrm{H}_{2} \mathrm{O}$ absorption coefficient at $296 \mathrm{~K}$, with a water mole fraction of $0.5 \%$, using data from the HITRAN database [29]. The inset shows the temporal LITGS signal (averaged over 500 laser shots) recorded at the peak of the water line at $3209.75 \mathrm{~cm}^{-1}$. The oscillation frequency is 11 $\mathrm{MHz}$ and the decay time is $0.58 \mu \mathrm{s}$.

The relative line intensities in Fig. 3 show some discrepancies between the simulation and the LITGS experiment. This can be attributed to absorption of the laser energy by water in the ambient air. As the laser is absorbed, the pump laser energy decreases over the path the laser travels before reaching the measurement volume. Thus, stronger water lines are excited by lower laser energy than weaker lines, which leads to a lower signal. The high signal-to-noise ratio indicates that LITGS can be used as a highly sensitive tool for trace amounts of water in gas flows. Moreover, the comparison of the temporally resolved LITGS signals in Figs. 2 and 3 reveals significant differences in the evolution of the signal. This is a result of the different energy transfer mechanisms between the excited molecules $\mathrm{C}_{2} \mathrm{H}_{4}$ and $\mathrm{H}_{2} \mathrm{O}$ with their 
environment, respectively. Hence, not only the spectral position but also the temporally resolved single-shot signal carries species-specific information.

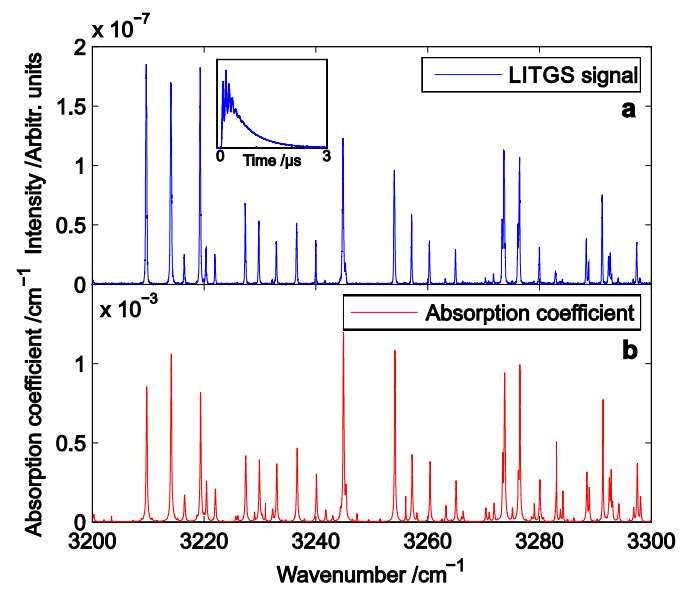

Figure 3. (a) LITGS excitation scan of water in the ambient air at 296 K. (b) The simulated absorption coefficient of $0.5 \%$ of water in air at $296 \mathrm{~K}$. Inset in (a): LITGS temporal shape of water in the ambient air at $296 \mathrm{~K}$ recorded at $3209.75 \mathrm{~cm}^{-1}$.

\subsection{LITGS in flames}

Figure 4a shows LITGS excitation scans performed in a rich $(\Phi=2.57)$ and a lean $(\Phi=0.82)$ $\mathrm{C}_{2} \mathrm{H}_{4} /$ air flame. The measurement volume was located at 7-mm height above the burner (HAB). The black curve shows the scan in the lean flame and the blue curve was recorded in the rich flame. The rich flame scan is plotted with an offset of $1 \times 10^{-9}$ (arbitrary units) for an improved readability.

The excitation scan in the rich flame shows a broad, continuous spectral structure, which decreases in intensity from 3200 to $3300 \mathrm{~cm}^{-1}$. The excitation scan in the lean flame, on the other hand, shows sharp spectral lines. These sharper lines are also visible in the rich flame, on top of the broad spectral feature. In order to analyze the spectrum, a comparison is made with simulations of the water absorption coefficient from the HITEMP database [30]. It shows that 
the sharp lines are hot water lines. The broad spectral feature in the rich flame can be ascribed to absorption by various hydrocarbons in the flame. The product zone of a rich $\mathrm{C}_{2} \mathrm{H}_{4} / \mathrm{air}$ flame contains a multitude of hydrocarbon species with absorption lines in this spectral range, and most hydrocarbon spectra have very high line density at flame temperature. The spectrum of the rich flame also shows sharp dips in the continuous spectral feature at certain wavenumbers. The comparison with HITRAN simulations shows that these dips correspond to the strong water lines at low temperature (296 K). A decrease in the pump laser intensity due to absorption by water in the ambient air leads to a lower signal at these points in the spectrum. However, note that the LITGS signal is still solely generated in the measurement volume. Only the overall intensity is affected by this absorption, but not the signal characteristics that are utilized in the evaluation.

Figures 4b-c shows the temporally resolved LITGS signal recorded in the lean and rich flames. Excitation was made at the peak of the hot water line at $3231 \mathrm{~cm}^{-1}$. Each curve is an average of 500 shots. The signals have a higher oscillation frequency and a faster decay time than the ones recorded in the cold flows. This reflects the higher sound speed and enhanced thermal diffusion at flame temperature.

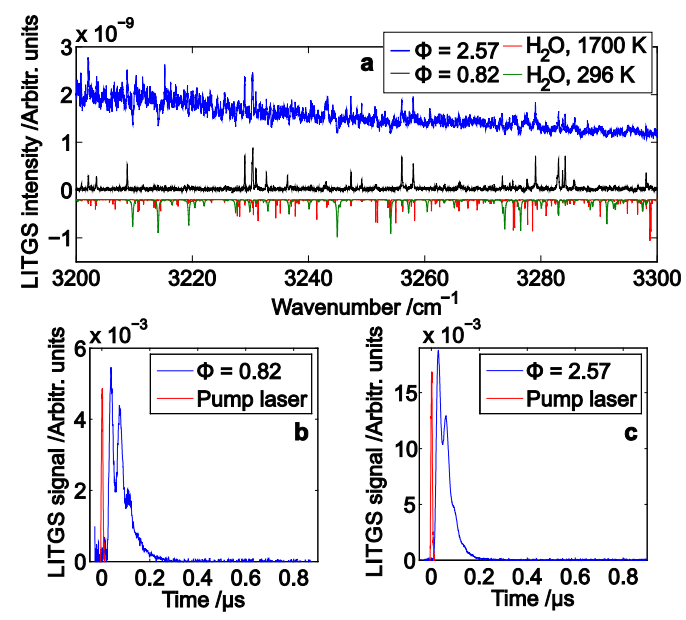

Figure 4. (a) LITGS excitation scan in $\mathrm{C}_{2} \mathrm{H}_{4}$ /air flames at $\Phi=2.57$ (blue) and $\Phi=0.82$ (black) compared with the simulated absorption coefficient of water at $1700 \mathrm{~K}$ (red) and $296 \mathrm{~K}$ (green). 
The relative intensity of the absorption coefficient has been adjusted to match the measurement. The lower figures show LITGS temporal shape recorded at $3231 \mathrm{~cm}^{-1}$ in the flame at (b) $\Phi=0.82$ and (c) $\Phi=2.57$.

In order to do a systematic assessment of the method, LITGS signals were recorded at $4 \mathrm{~mm}$ HAB for different equivalence ratios $\Phi$. Figure 5 a shows the oscillation frequency and decay time of the LITGS signals as a function of $\Phi$. The error bars indicate a $5 \%$ uncertainty in the oscillation frequency, as a result of the limited resolution in the Fourier transform, and a 10\% uncertainty in the decay time from the exponential fit.

Figure $5 b$ shows the flame temperature derived from the oscillation frequency using Eq. 1. Thermodynamics equilibrium calculations were performed to determine the adiabatic flame temperature $\left(T_{a d}\right)$ and the major species concentration in the flames at different $\Phi$. The measured temperature is significantly lower than the adiabatic temperature. This is attributed mainly to heat losses to the burner surface and to the bluff body stabilizer, which was placed quite close to the burner in order to stabilize the flames. The error bars, showing an uncertainty of $5-10 \%$, reflect both the uncertainty in the oscillation frequency and in the gas compositions. The gas composition is needed to determine the constants $\gamma / M_{\text {gas }}$ in Eq. 1. For lean and stoichiometric flames, the change in the gas constants with $\Phi$ is very small, as shown before [22]. For rich flames the gas constants were found to vary more significantly with gas composition. The most important influence in these changes was the $\mathrm{H}_{2}$ mole fraction in the flame. Thus, for accurate flame temperature measurements in rich flames using LITGS, accurate and precise determination of the $\mathrm{H}_{2}$ mole fraction is required. For most flames, however, reasonable estimates of the local hydrogen concentration are available in the literature. Future work will investigate the possibility of deriving both the temperature and the $\gamma / M_{\text {gas }}$ ratio simultaneously from measuring the oscillation frequency and the decay time. 

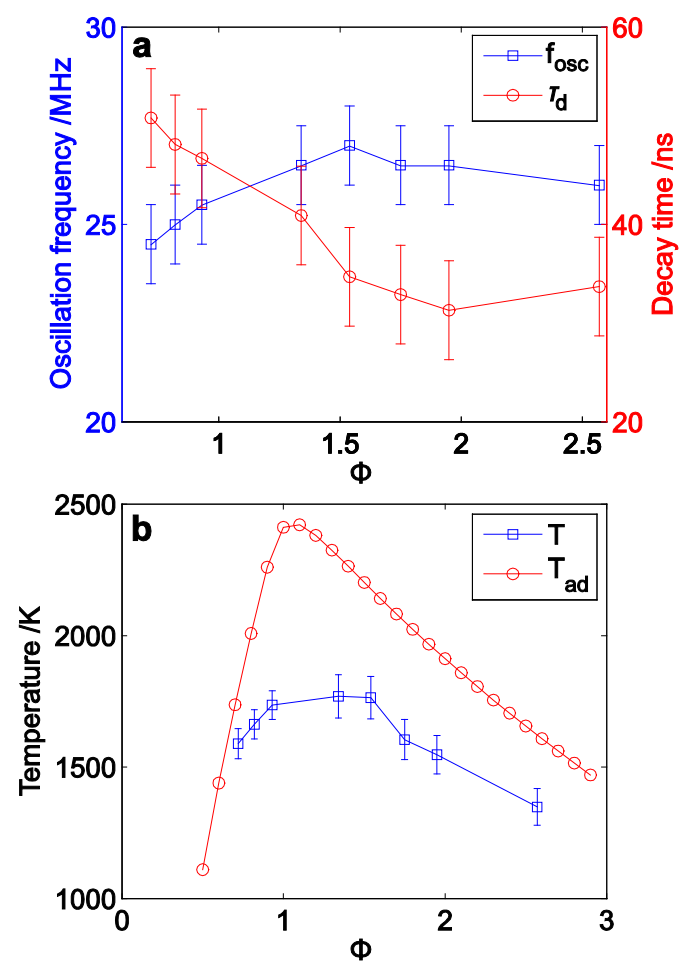

Figure 5: (a) Oscillation frequency and decay time of the LITGS signal in $\mathrm{C}_{2} \mathrm{H}_{4}$ /air flames with different $\Phi$. (b) Flame temperatures calculated from the LITGS oscillation frequencies for different $\Phi$ compared with the adiabatic flame temperature.

Figure 6 shows the time-resolved LITGS signals recorded at different HAB in the $\Phi=2.57$ flame. Each curve was recorded with excitation at $3231 \mathrm{~cm}^{-1}$ and is an average of 500 shots. The variations of the main characteristics between single shot signals were not significant, but the signal-to-noise ratio of the single shots was of the order of 20, hence the averaging. At some $\mathrm{HAB}$, there is a weak signal that appears simultaneously with the pump beams and disappears when at least one of the pump lasers is blocked. This is shown in the insets in Fig. 6. This signal occurs simultaneously with green fluorescence signals that can be observed along the probe beam path in the flame with the naked eye. Therefore it is likely that the additional signal is the result of a two-color four-wave mixing (FWM) process of a flame species, similar to the nonresonant-pump resonant-probe FWM of $\mathrm{OH}$ in a previous paper [31]. The probe laser at 457 
$\mathrm{nm}$ is in resonance with a weak line of the $\mathrm{C}_{2}$ Swan band. $\mathrm{C}_{2}$ generated in the reaction zone gives a weak signal at low HAB. Absorption of the pump lasers by the soot in the flame can generate $\mathrm{C}_{2}$ from the soot particles, which accounts for the stronger signal in the sooty region of the flame. Although not discussed, similar signals have previously been observed in LITGS measurements in sooty flames [21] when the probe laser was in resonance with another part of the $\mathrm{C}_{2}$ Swan band.
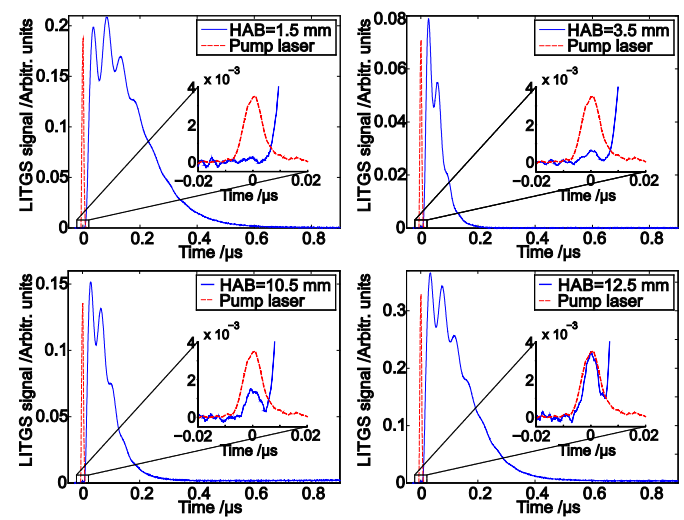

Figure 6. Time-resolved LITGS signals for different $H A B$ in the $\Phi=2.57$ flame. The red curve in the figures illustrates the timing of the mid-IR pump laser beams. The $C_{2}-F W M$ signal is shown in the inset in the plots.

It can also be seen in Fig. 6 that the decay time and oscillation frequency of the LITGS signals change with HAB. Figure 7a shows the oscillation frequency and the decay time vs. HAB in the $\Phi=2.57 \mathrm{C}_{2} \mathrm{H}_{4} /$ air flame. Close to the burner, the lower oscillation frequency and longer decay time indicates a slower sound speed and thermal diffusion in the gas suggesting comparatively low temperatures. Figure $7 \mathrm{~b}$ shows the temperature at different HAB calculated from the oscillation frequency. The calculations assume the gas composition is uniform throughout the probed volume in the flame. The temperature is lower close to the burner and 
close to the stabilizer, whereas in the middle it is quite constant within the measurement uncertainties. The qualitative temperature profile is in excellent agreement with those measured by other techniques in similar flames [5]. This highlights the potential of LITGS for combustion diagnostics.
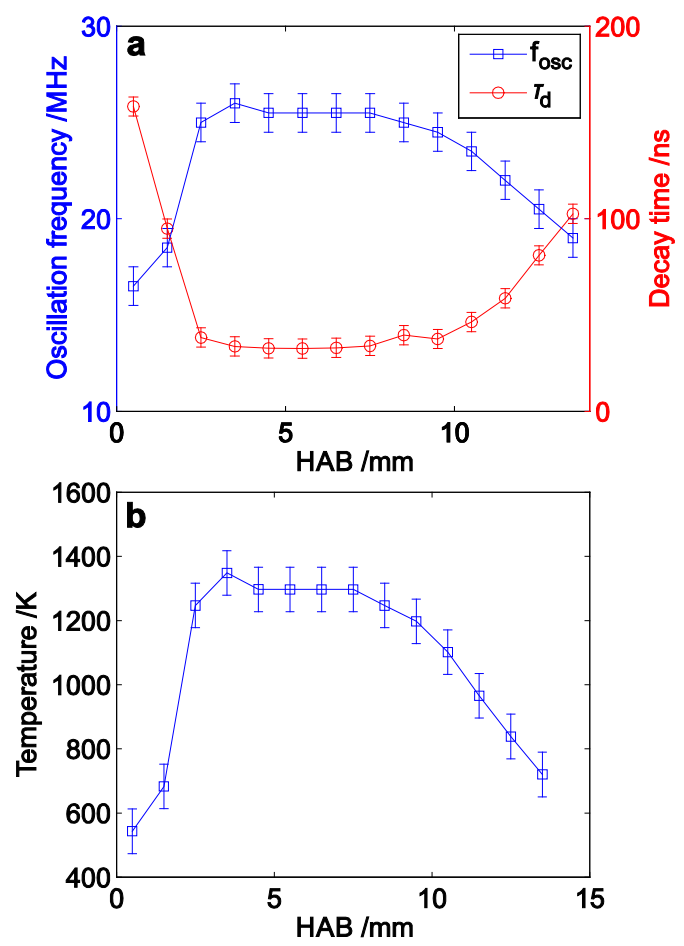

Figure 7: (a) Oscillation frequency and decay time of the LITGS signal at different HAB in the $\Phi=2.57$ flame. (b) Flame temperature calculated from the LITGS oscillation frequency at different $H A B$.

\section{Conclusions}

The application of mid-infrared LITGS to cold flows and flames was demonstrated probing strong infrared transitions involving fundamental molecular vibrations. The LITGS signal at room temperatures was found to be very sensitive to water in the ambient air. The infrared water 
absorption lines are very weak in the spectral region studied, but could still be detected with very good signal-to-noise ratio in the LITGS excitation scans.

Time-resolved LITGS measurements were performed in lean and rich $\mathrm{C}_{2} \mathrm{H}_{4} /$ air flames. Clear signals could be detected even in sooty flames at $\Phi=2.57$ with minimal interference from scattering. The LITGS signal can be recorded both as a temporally resolved signal at one wavelength, or the LITGS intensity can be recorded as function of wavelength to yield the spectrum. Excitation scans at room temperature show sharp spectral features that agree well with the absorption cross section simulations. The spectrum in lean flames shows sharp spectral features of hot water. In rich flames, the broad spectral features from hot hydrocarbon species dominate the spectrum and result in high signal levels. On the other hand, the temporally resolved LITGS signal contains a lot of information offering the potential for single-shot multiparameter measurements. Molecular energy transfer rates, speed of sound, and thermal diffusion in the medium can be determined simultaneously and provide information about the gas composition and the temperature. In particular, the hydrogen concentration may be measureable as the amount of hydrogen has the strongest effects on the gas properties.

In addition, in the rich flame our approach allowed the observation of a two-color FWM signal in the temporally resolved LITGS traces. This occurs from a resonance of the probe beam with $\mathrm{C}_{2}$ radicals, causing a non-resonant-pump resonant-probe interaction of the pump and probe beams. Consequently, the $\mathrm{C}_{2}$ distribution can be determined without an additional experimental effort.

Overall, our work demonstrates that IR-LITGS is a versatile tool for combustion diagnostics. Particularly interesting and promising future applications seem to be in the analysis of sooty flames, where established methods often reach their limits due to the presence of soot precursors and particles. LITGS may become a true alternative means of diagnostics as the soot nuclei and 
particles can result in characteristic modifications in the signal and thus allow additional information to be gained.

\section{Acknowledgement}

We thank for the support from the Swedish Energy Agency, the Knut \& Alice Wallenberg foundation, the Swedish Research Council (VR), the European Research Council (ERC) Advanced Grant TUCLA, and the LASERLAB-EUROPE (grant agreement no. 284464, EC's Seventh Framework Programme). 
References

[1] R.S. Barlow, Proc. Combust. Inst., 31 (2007) 49-75.

[2] S. Roy, J.R. Gord, A.K. Patnaik, Prog. Energy Combust. Sci., 36 (2010) 280-306.

[3] J. Kiefer, P. Ewart, Prog. Energy Combust. Sci., 37 (2011) 525-564.

[4] C.J. Kliewer, Y. Gao, T. Seeger, J. Kiefer, B.D. Patterson, T.B. Settersten, Proc. Combust. Inst., 33 (2011) 831-838.

[5] C. Brackmann, J. Bood, P.-E. Bengtsson, T. Seeger, M. Schenk, A. Leipertz, Appl. Opt., $41(2002)$ 564-572.

[6] R.L. Farrow, R.P. Lucht, W.L. Flower, R.E. Palmer, Proc. Combust. Inst., 20 (1984) 1307-1312.

[7] S.P. Kearney, K. Frederickson, T.W. Grasser, Proc. Combust. Inst., 32 (2009) 871-878.

[8] S.P. Kearney, M.N. Jackson, AIAA J., 45 (2007) 2947-2956.

[9] A. Braeuer, F. Beyrau, M.C. Weikl, T. Seeger, J. Kiefer, A. Leipertz, A. Holzwarth, A. Soika, J. Raman Spectrosc., 37 (2006) 633-640.

[10] M. Aldén, S. Wallin, Appl. Opt., 24 (1985) 3434-3437.

[11] S. Williams, L.A. Rahn, P.H. Paul, J.W. Forsman, R.N. Zare, Opt. Lett., 19 (1994) 16811683.

[12] J.T. Fourkas, T.R. Brewer, H. Kim, M.D. Fayer, J. Chem. Phys., 95 (1991) 5775-5784.

[13] A. Hell, F. Förster, B. Weigand, J. Raman Spectrosc., in print (2016).

[14] H. Latzel, A. Dreizler, T. Dreier, J. Heinze, M. Dillmann, W. Stricker, G.M. Lloyd, P. Ewart, Appl. Phys. B: Lasers Opt., 67 (1998) 667-673.

[15] R.C. Hart, R.J. Balla, G.C. Herring, Meas. Sci. Technol., 8 (1997) 917.

[16] B. Hemmerling, D.N. Kozlov, O.M. Stel'makh, B. Attal-Tretout, Chem. Phys., 320 (2006) 103-117. 
[17] A. Stampanoni-Panariello, B. Hemmerling, W. Hubschmid, Appl. Phys. B: Lasers Opt., 67 (1998) 125-130.

[18] B. Hemmerling, D.N. Kozlov, Appl. Opt., 38 (1999) 1001-1007.

[19] M. Gutfleisch, D.I. Shin, T. Dreier, P.M. Danehy, Appl. Phys. B: Lasers Opt., 71 (2000) 673-680.

[20] B. Hemmerling, A. Stampanoni-Panariello, Appl. Phys. B: Photophys. Laser Chem., 57 (1993) 281-285.

[21] M.S. Brown, W.L. Roberts, J. Propul. Power, 15 (1999) 119-127.

[22] M.S. Brown, Y. Li, W.L. Roberts, J.R. Gord, Appl. Opt., 42 (2003) 566-578.

[23] B. Williams, M. Edwards, R. Stone, J. Williams, P. Ewart, Combust. Flame, 161 (2014) 270-279.

[24] A.L. Sahlberg, J. Kiefer, M. Aldén, Z.S. Li, Appl. Spectrosc., in print (2015).

[25] Z.S. Li, C.H. Hu, J. Zetterberg, M. Linvin, M. Aldén, J. Chem. Phys., 127 (2007) 084310-084310.

[26] Z.S. Li, M. Rupinski, J. Zetterberg, Z.T. Alwahabi, M. Aldén, Chem. Phys. Lett., 407 (2005) 243-248.

[27] A. Stampanoni-Panariello, D.N. Kozlov, P.P. Radi, B. Hemmerling, Appl. Phys. B: Lasers Opt., 81 (2005) 101-111.

[28] A. Stampanoni-Panariello, D.N. Kozlov, P.P. Radi, B. Hemmerling, Appl. Phys. B: Lasers Opt., 81 (2005) 113-129.

[29] L.S. Rothman, I.E. Gordon, Y. Babikov, A. Barbe, D. Chris Benner, P.F. Bernath, M. Birk, L. Bizzocchi, V. Boudon, L.R. Brown, A. Campargue, K. Chance, E.A. Cohen, L.H. Coudert, V.M. Devi, B.J. Drouin, A. Fayt, J.-M. Flaud, R.R. Gamache, J.J. Harrison, J.M. Hartmann, C. Hill, J.T. Hodges, D. Jacquemart, A. Jolly, J. Lamouroux, R.J. Le Roy, G. Li, D.A. Long, O.M. Lyulin, C.J. Mackie, S.T. Massie, S. Mikhailenko, H.S.P. Müller, O.V. Naumenko, A.V. Nikitin, J. Orphal, V. Perevalov, A. Perrin, E.R. 
Polovtseva, C. Richard, M.A.H. Smith, E. Starikova, K. Sung, S. Tashkun, J. Tennyson, G.C. Toon, V.G. Tyuterev, G. Wagner, J. Quant. Spectrosc. Radiat. Transfer., 130 (2013) 4-50.

[30] L.S. Rothman, I.E. Gordon, R.J. Barber, H. Dothe, R.R. Gamache, A. Goldman, V.I. Perevalov, S.A. Tashkun, J. Tennyson, J. Quant. Spectrosc. Radiat. Transfer, 111 (2010) 2139-2150.

[31] Z.W. Sun, J. Kiefer, Z.S. Li, B. Li, M. Alden, Appl. Phys. B: Lasers Opt., 92 (2008) 287-293. 